annrheumdis-2019-216150

\title{
Neuropsychiatric Events in Systemic Lupus Erythematosus
}

\section{A longitudinal analysis of outcomes in an international Inception Cohort using a Multistate Model approach}

John G. Hanly MD${ }^{1}$, Murray B. Urowitz $\mathrm{MD}^{2}$, Caroline Gordon $\mathrm{MD}^{3}$, Sang-Cheol Bae MD PhD , Juanita Romero-Diaz MD MSc ${ }^{5}$, Jorge Sanchez-Guerrero MD MSc², Sasha Bernatsky MD PhD ${ }^{6}$, Ann E. Clarke MD MSc ${ }^{7}$, Daniel J Wallace $\mathrm{MD}^{8}$, David A. Isenberg $\mathrm{MD}^{9}$, Anisur Rahman MD $\mathrm{PhD}^{9}$, Joan T. Merrill $M D^{10}$, Paul R. Fortin MD MPH ${ }^{11}$, Dafna D. Gladman $\mathrm{MD}^{2}$, lan N. Bruce MD ${ }^{12}$, Michelle Petri MD MPH ${ }^{13}$, Ellen M. Ginzler MD MPH ${ }^{14}$, M.A. Dooley MD MPH ${ }^{15}$, Rosalind Ramsey-Goldman MD DrPH ${ }^{16}$, Susan Manzi MD MPH ${ }^{17}$, Andreas Jonsen MD $\mathrm{PhD}^{18}$, Graciela S. Alarcón MD MPH ${ }^{19}$, Ronald F. van Vollenhoven $\mathrm{MD}^{20}$, Cynthia Aranow $\mathrm{MD}^{21}$, Meggan Mackay $\mathrm{MD}^{21}$, Guillermo Ruiz-Irastorza $\mathrm{MD}^{22}, \mathrm{~S}$. Sam Lim MD $\mathrm{MPH}^{23}$, Murat Inanc $\mathrm{MD}^{24}$, Kenneth C. Kalunian $\mathrm{MD}^{25}$, Soren Jacobsen MD DMSc${ }^{26}$, Christine A. Peschken $\mathrm{MD}^{27}$, Diane L. Kamen $\mathrm{MD}^{28}$, Anca Askanase MD MPH${ }^{29}$, Vernon Farewell $\mathrm{PhD}^{30}$

\section{Institutions:}

1Division of Rheumatology, Department of Medicine and Department of Pathology, Queen Elizabeth II Health Sciences Centre and Dalhousie University, Halifax, Nova Scotia, Canada;

${ }^{2}$ Centre for Prognosis Studies in the Rheumatic Diseases, Toronto Western Hospital and University of Toronto, ON, Canada;

${ }^{3}$ Rheumatology Research Group, Institute of Inflammation and Ageing, College of Medical and Dental Sciences, University of Birmingham, Birmingham, UK; 
${ }^{4}$ Department of Rheumatology, Hanyang University Hospital for Rheumatic Diseases, Seoul, Korea;

${ }^{5}$ Instituto Nacional de Ciencias Médicas y Nutrición, Mexico City, Mexico;

${ }^{6}$ Divisions of Rheumatology and Clinical Epidemiology, Department of Medicine, McGill University, Montreal, Quebec, Canada;

${ }^{7}$ Division of Rheumatology, Cumming School of Medicine, University of Calgary, Calgary, Alberta, Canada;

${ }^{8}$ Cedars-Sinai/David Geffen School of Medicine at UCLA, Los Angeles, CA, USA;

${ }^{9}$ Centre for Rheumatology, Department of Medicine, University College London, UK;

${ }^{10}$ Department of Clinical Pharmacology, Oklahoma Medical Research Foundation, Oklahoma City, OK, USA;

${ }^{11}$ Division of Rheumatology, Department of Medicine, CHU de Québec, Université Laval, Quebec City, Canada;

${ }^{12}$ Arthritis Research UK Epidemiology Unit, Faculty of Biology Medicine and Health, Manchester Academic Health Sciences Centre, The University of Manchester, and NIHR Manchester Musculoskeletal Biomedical Research Centre, Manchester University NHS Foundation Trust, Manchester Academic Health Science Centre Manchester, UK;

${ }^{13}$ Division of Rheumatology, Johns Hopkins University School of Medicine, Baltimore, MD, USA;

${ }^{14}$ Department of Medicine, SUNY Downstate Medical Centre, Brooklyn, NY, USA;

${ }^{15}$ Thurston Arthritis Research Centre, University of North Carolina, Chapel Hill, NC, USA; 
${ }^{16}$ Northwestern University and Feinberg School of Medicine, Chicago, IL, USA;

${ }^{17}$ Lupus Centre of Excellence, Allegheny Health Network, Pittsburgh, PA, USA;

${ }^{18}$ Department of Clinical Sciences Lund, Rheumatology, Lund University, Lund, Sweden.

${ }^{19}$ Department of Medicine, University of Alabama at Birmingham, Birmingham, AL, USA;

${ }^{20}$ Department of Rheumatology and Clinical Immunology, Amsterdam University Medical Centres, Amsterdam, Holland.

${ }^{21}$ Feinstein Institute for Medical Research, Manhasset, NY, USA;

${ }^{22}$ Autoimmune Diseases Research Unit, Department of Internal Medicine, BioCruces Health Research Institute, Hospital Universitario Cruces, University of the Basque Country, Barakaldo, Spain;

${ }^{23}$ Emory University, Department of Medicine, Division of Rheumatology, Atlanta, Georgia, USA

${ }^{24}$ Division of Rheumatology, Department of Internal Medicine, Istanbul Medical Faculty, Istanbul University, Istanbul, Turkey;

${ }^{25}$ UCSD School of Medicine, La Jolla, CA, USA;

${ }^{26}$ Copenhagen Lupus and Vasculitis Clinic, 4242, Rigshospitalet, Copenhagen University Hospital, Copenhagen, Denmark;

${ }^{27}$ University of Manitoba, Winnipeg, Manitoba, Canada;

${ }^{28}$ Medical University of South Carolina, Charleston, South Carolina, USA;

${ }^{29}$ Hospital for Joint Diseases, NYU, Seligman Centre for Advanced Therapeutics, New York NY; 
${ }^{30} \mathrm{MRC}$ Biostatistics Unit, Cambridge Institute of Public Health, University of Cambridge, Cambridge, UK;

Address Correspondence and Reprints to: Dr. John G Hanly, Division of Rheumatology, Nova Scotia Rehabilitation Centre (2 $2^{\text {nd }}$ Floor), 1341 Summer Street, Halifax, Nova Scotia, Canada, B3H 4K4.

Telephone: (902) 473 3818; $\quad$ Fax: (902) 4737019 ;

Email: john.hanly@nshealth.ca

Abstract word count: 250/250

Manuscript word count: 2,972/3,000 


\section{Key Messages}

\section{What is already known about this subject?}

$>$ Involvement of the nervous system in SLE is well recognized but the frequency and outcomes have not been well documented in different stages of the disease.

\section{What does this study add?}

All neuropsychiatric (NP) events were documented over a mean follow-up of 7.6 years in a large observational inception cohort study of SLE patients.

$>$ A multistate modelling approach was used to describe the frequency, attribution, association with health-related quality of life and clinical outcome of NP events.

> Predictive probability models were derived to estimate the likelihood of changing NP states over the first 10 years of SLE.

\section{How might this impact on clinical practice or future developments?}

$>$ Most NP events in SLE patients occur early in the disease course, have a negative impact on health-related quality of life and are attributed to SLE in $30 \%$ of cases.

Multistate modelling is well suited to the study of NPSLE and could serve as an outcome measure in clinical trials (e.g. comparing the rate of transition between NPSLE states for patients receiving active treatment and comparator; duration of time spent in different NPSLE states over the course of study). It also provides the basis for economic studies of health care costs in SLE patients with NP events, either attributed to SLE or non-SLE causes. 
Objectives: Using a reversible multistate model, we prospectively examined neuropsychiatric (NP) events for attribution, outcome and association with health-related quality of life (HRQoL), in an international, inception cohort of SLE patients.

Methods: Annual assessments for 19 NP events attributed to SLE and non-SLE causes, physician determination of outcome and patient HRQoL (SF-36 scores) were measured. Time-to-event analysis and multistate modelling examined the onset, recurrence and transition between NP states.

Results: NP events occurred in 955/1,827 (52.3\%) patients and 592/1910 (31.0\%) unique events were attributed to SLE. In the first two years of follow-up the relative risk $(95 \% \mathrm{Cl})$ for SLE NP events was $6.16(4.96,7.66)$ and non-SLE events was $4.66(4.01,5.43)$ compared with thereafter. Patients without SLE NP events at initial assessment had a $74 \%$ probability of being event-free at 10 years. For nonSLE NP events the estimate was $48 \%$. The majority of NP events resolved over 10 years but mortality was higher in patients with NP events attributed to SLE (16\%) versus patients with no SLE events $(6 \%)$ while the rate was comparable in patients with non-SLE NP events (7\%) compared to patients with no non-SLE events (6\%). Patients with NP events had lower SF-36 summary scores compared to those without NP events and resolved NP states $(p<0.001)$.

Conclusions: NP events occur most frequently around the diagnosis of SLE. Although the majority of events resolve they are associated with reduced HRQoL and excess mortality. Multistate modelling is well suited for the assessment of NP events in SLE.

Key words: Systemic lupus erythematosus, Multistate modeling, Neuropsychiatric 
Nervous system disease in SLE consists of neurologic and psychiatric events, predominantly affecting the central nervous system(1). Neuropsychiatric (NP) events vary in frequency, complexity, time of onset, rates of resolution and recurrence. Approximately $30 \%$ of NP events are attributed to $\operatorname{SLE}(2,3)$, although the rate varies between individual manifestations. Regardless of attribution, the majority of events are associated with lower self-reported health related quality of life (HRQoL)(2).

There are few clinical trials to guide interventions in SLE patients with NP events. Many observational studies are single centre experiences, frequently cross-sectional in design and utilize prevalent SLE cohorts. Longitudinal studies have not captured the bidirectional movement of patients between remissions and relapses of NP events, the duration in different NP states and likelihood of moving from one state to another over time.

Multistate models(4) offer a convenient and flexible framework to characterize changes in NP disease and provide a dynamic representation of the disease in continuous time. They also estimate time spent in different states and probabilities of being in particular states following specified time periods. Such summary inferences are more informative than models that focus on single, often dichotomous, outcomes such as the time to a specific clinical event. In common with other time-to-event modelling, these inferences are based on all follow-up data, not simply on data from subsets of patients with a specific follow-up time.

The current study utilized data from a large, prospective, international, disease inception cohort of SLE patients, who underwent annual assessment for NP events for up to 18 years. The overall objective 
was to model, over time, patient status with respect to NP events, incorporating attribution and association with HRQoL. To capture dynamic change in NP events, we adopted a reversible multistate model characterized by transition rates between states.

\section{Patients and Methods}

Research study network: The study was conducted by the Systemic Lupus International Collaborating Clinics (SLICC)(5), a network of 52 investigators at 43 academic centres in 16 countries. Recently diagnosed SLE patients were recruited from 31 SLICC sites in Europe, Asia, and North America. Data were collected per protocol at enrollment and annually ensuring data quality, management and security. The Nova Scotia Health Authority central zone Research Ethics Board and each of the participating centres' research ethics review boards approved the study. This research was planned without patient involvement.

Patients: Enrollment was permitted up to 15 months following diagnosis of SLE, taken as when the revised ACR classification criteria(6) were first recognized. Lupus-related variables included the SLE Disease Activity Index 2000 (SLEDAI-2K)(7) and SLICC/ACR Damage Index (SDI)(8).

Neuropsychiatric events: NP events were characterized within an enrollment window (6 months prior to the diagnosis of SLE up to the enrollment date) using ACR case definitions for 19 NP syndromes(9). Patients were reassessed annually within a 6-month window using a detailed protocol to record information on 19 NP syndromes(9), presence of pre-specified non-SLE causes, results of appropriate 
investigations, medications and outcomes. New NP events and status of previous NP events were determined at each assessment. For recurring events within an assessment period, the date of the first episode was taken as the onset of the event.

Attribution of NP events: Factors considered in the attribution decision rules included: (i) temporal onset of NP event(s) in relation to the diagnosis of SLE; (ii) concurrent non-SLE factor(s), such as potential causes ("exclusions") or contributing factors ("associations") for each NP syndrome in the glossary for the ACR case definitions of NP events(9); and (iii) "common" NP events that are frequent in normal population controls as described by Ainiala et al(10). These include isolated headaches, anxiety, mild depression (mood disorders failing to meet criteria for "major depressive-like episodes"), mild cognitive impairment (deficits in less than three of the eight specified cognitive domains) and peripheral neuropathy without electrophysiological confirmation. Two attribution decision rules of different stringency (models A and B) were derived $(11,12)$.

Attribution Model A (more stringent): NP events attributed to SLE (i) had their onset within the enrollment window or subsequently and (ii) had no "exclusions" or "associations" and (iii) were not one of the NP events identified by Ainiala(10).

Attribution Model B (less stringent): NP events attributed to SLE (i) had their onset within 10 years of the diagnosis of SLE and were still present within the enrollment window, or occurred subsequently, and (ii) had no "exclusions" and (iii) were not one of the NP events identified by Ainiala(10).

All NP events attributed to SLE using model A were included in the NP events using model B. All other events were classified as a non-SLE NP event. 
Outcome of NP events: Physician-generated 7-point Likert scale score at each follow-up assessment compared the change in NP events between onset and follow-up (1=patient demise, 2=much worse, $3=$ worse, $4=$ no change, $5=$ =improved, $6=$ much improved, $7=$ resolved)(13). Separately a patientgenerated SF-36 questionnaire at each assessment provided eight subscale scores and mental (MCS) and physical (PCS) component summary scores $(13,14)$.

Statistical analysis: Two multistate patient level models were examined (Figure 1), one for NP events attributed to SLE(model B) and the other for non-SLE events. Non-SLE events were ignored during modelling of SLE events and vice-versa. The four states were:

1. No NP event ever..

2. No current NP event but $\geq 1$ in the past. State entry was the time of resolution of NP event(s).

3. New/Ongoing NP event(s) with state entryat onset of NP event.

4. Death

Modelling assumed transitions occurred at any time, not just at assessments. Each site investigator provided the dates for onset and resolution of NP events and precise dates for death.

The time origin was 6 months before SLE diagnosis. The transition rate which characterizes the probability of changing from State 1 to State 3 in the first two years of follow-up was allowed to differ from the rate thereafter, as many events occurred in the earlier time period. All other transition rates were assumed constant.. Patients could move back and forth between States 2 (resolution of NP event) and 3 (new/ongoing NP event). The death rate was assumed to be the same from States 2 and 3 but a separate rate was allowed from State 1 (no history of NP events). The model can be extended to 
allow explanatory variables to influence transition rates through a regression model on the logarithm of the transition rates. Maximum likelihood estimation of the model was implemented using the $R(15)$ package 'msm'(16). SF-36 analyses used linear regression models with robust estimation via generalized estimating equations to adjust for correlation between multiple measurements for the same patient.

\section{Results}

Patients: 1,827 patients were recruited from October 1999 through December 2011, from the United States [n=540 (29.5\%)], Europe [n=477 (26.1\%)], Canada [n=418 (22.9\%)], Mexico [n=223 (12.2\%)] and Asia [n=169 (9.3\%)]. At enrollment, the mean (SD) age was 35.1 (13.3) years, $88.8 \%$ of patients were female, with variable race/ethnicity (Caucasian 48.8\%, African 16.8\%, Hispanic 15.4\%, Asian $15.1 \%$ and other $3.9 \%)$ and the mean (SD) disease duration was 5.6 (4.2) months. The mean (SD) SLEDAI-2K was 5.3 (5.4) and SDI was 0.32 (0.74). Medications at enrollment included corticosteroids (70.3\%), antimalarials $(67.4 \%)$, immunosuppressants $(40.1 \%)$, warfarin $(5.4 \%)$, low dose aspirin (14.3\%), antidepressants (10.1\%), anticonvulsants (4.4\%) and antipsychotic drugs (0.7\%). The mean follow-up was $7.6 \pm 4.6$ years, with1 to 19 assessments and ended in September 2017. One hundred patients died during the study.

Neuropsychiatric manifestations: NP events occurred in 955/1,827 (52.3\%) patients and 493/1,827 $(27.0 \%)$ had $\geq 2$ events. There were 1,910 unique NP events, encompassing all 19 NP syndromes(9), of which $1,749(91.6 \%)$ involved the CNS and $161(8.4 \%)$ the peripheral nervous system (PNS)(9). The NP events attributed to SLE varied from 17.9\% (attribution model A) to $31.0 \%$ (attribution model B) and 
occurred in $13.5 \%$ (model A) to $21.2 \%$ (model B) of patients. Details are provided in supplementary Tables S1 and S2.

Transition rates between NP states, duration spent in each state and time to event analysis: The number of observed changes between NP states, for SLE and non-SLE events (Table 1) provide the basis for estimation of the multistate models. 
Table 1: Observed changes between neuropsychiatric (NP) states and death for patients with SLE NP events and non-SLE NP events as determined using attribution model $B$. The lower part of the table shows the estimated average $(95 \% \mathrm{Cl})$ of total time (years) spent in neuropsychiatric (NP) and death states over 10 years of follow-up after cohort entry.

\section{Transition to state}

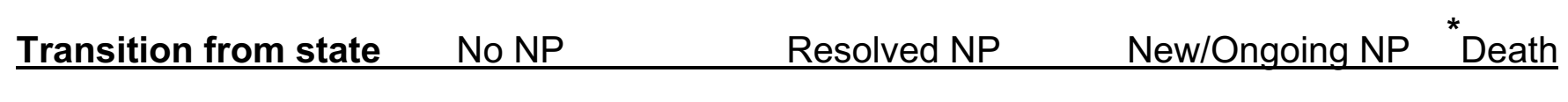

\section{SLE NP events}

No NP

12,539

-

387

61

Resolved NP

1,561

60

18

New/Ongoing NP

270

1,541

21

\section{Non-SLE NP events}

No NP

8,782

$-$

810

66

Resolved NP

2,603

224

13

New/Ongoing NP

645

3,294

21

Estimated time (years) spent in state

\section{SLE NP}

$8.81 \quad 0.39$

$(8.70,8.92)$

Non-SLE NP
7.32

$(7.14,7.48)$
$(0.33,0.44)$

0.92

$(0.84,1.01)$
0.56

0.24

$(0.21,0.32)$

$(0.50,0.64)$

1.46

0.30

$(1.36,1.58)$

\footnotetext{
* For the lower portion of Table 1, this refers to the time following death, i.e. in the death state, over the 10 years of observation.
} 
Some patients remained in the same NP state and others moved through one or more states. The table also summarizes the estimated average time spent in NP states or death state over 10 years of followup, assuming all patients were in the no NP event state initially. A composite of NP state occupancy and duration is illustrated in lasagna plots (Figure 2) where trajectories are displayed as a layered plot. For NP events attributed to SLE, the time spent in a new/ongoing NP state was lower (0.56) and the time spent in the no NP state was higher (8.81) than for NP events attributed to non-SLE causes (1.46 for new/ongoing state and 7.32 for the no NP state).

The time to onset of new and recurrent NP events is illustrated in Figure 3. For recurrent events the time origin was the resolution of any previous event. The rate of occurrence of first NP events was highest in the early years following the diagnosis of SLE, consistent with our modelling strategy of allowing a differential rate of events in the first two years of follow-up. Based on the multistate models, the estimated relative risk $[95 \%$ confidence interval $(\mathrm{Cl})]$ in the first two years compared with the subsequent time period, was $6.16(4.96,7.66)$ for SLE NP events and $4.66(4.01,5.43)$ for non-SLE events. Recurrent SLE NP events occurred at a higher rate than first events with little difference in these rates for non-SLE events.

Predictive probabilities for transitioning between NP states over time: The same multistate models provide estimates of the probability of having changed NP states or died over defined periods of time. The estimates for 10 years after entry into the no NP, resolved NP or new/ongoing NP event states are summarized in Table 2. 
Table 2: Estimated predictive probabilities of the state a patient will be in after 10 years of follow-up for the three possible initial states, tabulated separately for SLE NP events and non-SLE NP events as determined using attribution model $\mathrm{B}$.

\section{Estimated probability $(95 \% \mathrm{Cl})$ of being in NP state}

Initial NP state

No NP

SLE NP events

Non-SLE NP events
0.13

$(0.12,0.15)$

0.26

$(0.24,0.28)$
New/Ongoing NP Death

$(0.72,0.76)$

0.48

$(0.46,0.51)$

Resolved NP

No NP Resolved NP New/O

\section{Resolved NP}

SLE NP events

0.72

0.13

0.16

$(0.68,0.74)$

0.67

$(0.10,0.16)$

$(0.12,0.21)$

Non-SLE NP events

$(0.64,0.70)$

0.26

0.07

$(0.23,0.29)$

$(0.05,0.09)$

\section{New/Ongoing NP}

SLE NP events

Non-SLE NP events
0.64

$(0.61,0.68)$

0.62

$(0.59,0.65)$
0.20

0.16

$(0.17,0.24) \quad(0.12,0.21)$

0.31

0.07

$(0.28,0.34) \quad(0.05,0.09)$ 
For patients with no SLE NP event at cohort entry, there was an estimated $74 \%$ probability of remaining free of such events 10 years later. For NP events not attributed to SLE the estimate was $48 \%$. For patients with resolved or new/onging NP events, the majority would be in a resolved NP state after 10 years. Estimates of having new/ongoing NP events after 10 years of follow-up were $13 \%$ and $20 \%$ for SLE NP events if the patient started follow-up in the resolved and new/ongoing states, respectively, and $26 \%$ and $31 \%$ for non-SLE NP events. Of note, the estimates of dying in a 10 year period were higher in patients with NP events (new/ongoing or resolved) attributed to SLE (16\%) versus patients without SLE NP events $(6 \%)$ with an estimated relative risk $(\mathrm{RR}, 95 \% \mathrm{Cl})$ of death of $4.3(2.7,6.7)$. Patients with non-SLE NP events had a lower death rate $(7 \%)$, similar to patients with no non-SLE events $(6 \%)$, with an estimated RR of $1.3(0.8,2.0)$. After adjustment for age and post-secondary education, the RR associated with SLE NP events was slightly lower $[R R=2.9(1.6,5.6)]$ but still substantially higher than that for non-SLE events $[R R=1.3(0.7,2.4)]$.

A comprehensive investigation of predictors for onset and resolution of NP events at the patient level is beyond the scope of this paper. Preliminary analyses in supplementary Tables S3 and S4 provide RR estimates, based on a multistate model using multivariate regression for predictors at cohort entry. The effects are assumed to be the same on the transitions to the NP event state from both the no NP event and the resolved state. The results suggest that males have higher rates of onset and resolution for both types of NP events. Asian race and post-secondary education are protective for SLE NP event onset and Asian and Hispanic race are associated with somewhat higher rates of resolution for 
SLE events. Hispanic race and a higher age at diagnosis is protective for the onset of non-SLE NP events while all other races have a higher resolution rate for these events than Caucasians. Higher age at diagnosis is linked to a lower resolution rate.

The association between NP states and health-related quality of life: Patient generated PCS and MCS scores associated with different NP states are summarized in Figure 4. There were clinically lower PCS and MCS scores in the new/ongoing NP state compared to the no NP and resolved NP states (global $p$-value<0.001). This was true for patient states, which could vary over time, defined by both SLE and non-SLE NP events.

\section{Discussion}

Heterogeneity of clinical manifestations, uncertainty in causal attribution, incomplete understanding of pathogenesis and few controlled clinical trials to guide treatment contribute to the challenge of NPSLE. Long-term studies of representative SLE cohorts provide information on the clinical course with current standard of care. A multistate modelling approach determined the frequency of NP events, their clinical outcomes and impact on HRQoL in an international prospective cohort of SLE patients. Estimates for occurrence and resolution of NP events over time in patients receiving standard of care provides a benchmark for clinical trials of new therapies. 
The SLICC inception cohort has information on the occurrence and outcome of individual NP manifestations in addition to other SLE manifestations, comorbidities and treatment. Our study captures the totality of nervous system events and attribution over a mean follow-up of 7.6 years. Major organ manifestations of SLE frequently present early in the disease course, and thus a disease inception cohort is advantageous. Additional benefits include standardized, comprehensive assessments for NP events and centralized data driven decisions on attribution to SLE and non-SLE causes. The attribution of up to $30 \%$ of NP events to SLE in up to $20 \%$ of SLE patients is consistent with previous SLICC cohort studies $(12,17)$ and with other recent studies $(3,18)$.

Multistate modelling has been applied to psoriatic arthritis $(19,20)$ lupus nephritis $(21)$ and organ damage accrual in SLE(22) but has not previously been used to study NPSLE. Although NP events can present or recur at any time in the disease course, they are most frequent in the first few years following SLE diagnosis. This is the case for both NP events attributed to SLE and non-SLE causes, although the rate of recurrence compared to the rate for the initial event is higher for NP events attributed to SLE. As is the case with other SLE manifestations $(21,23)$, the occurrence of NP manifestations has implications for the subsequent disease course. Patients whose initial state was free of SLE NP events, had a $74 \%$ likelihood of being free of SLE NP events at 10 years of follow-up. For patients still free of SLE NP events two years after the diagnosis, this probability rises to $84 \%$. Thus, if patients remain event free during this time, there is a high likelihood that NPSLE manifestations will not occur subsequently. For patients whose initial SLE NP state was 
"resolved", or who subsequently had all NP events resolve, there was a $72 \%$ probability of being free of SLE NP events after 10 years of follow-up.

Individual types of NP events, regardless of attribution, are associated with reduced HRQoL $(12,13,17)$ that is clinically and statistically significant. The current study found a similar association between NP events in total and reduced HRQoL that reverts towards normal with resolution of the events. More ominously, following 10 years of follow-up, there is a higher probability $(16 \%)$ of death for patients who experience SLE NP events compared to those without SLE NP events $(6 \%)$ with little increase associated with nonSLE NP events (7\%). Another recent large, long-term study(3) found that patients with NPSLE had a three-fold higher mortality with a hazard ratio $(95 \% \mathrm{Cl})$ of 3.09 (0.03-9.21). Thus, major organ involvement by SLE carries a higher mortality risk over time, although the cause of death is not necessarily attributed to affected organ systems(23).

Transition rates between NP states were derived from clinically meaningful changes identified by treating physicians and supported by patient self-report health status. Potential applications of multistate modelling include using transition rates as a primary outcome in clinical trials and projecting the cost of care for NP disease. For example, the estimated probability of an SLE NP event resolving within 2 years is 0.31 . A clinical trial to detect a $50 \%$ improvement in that rate would require a sample size (with an alpha level of 0.05 and $80 \%$ power) of 282 patients (141/group). Furthermore, by determining the 
actual costs for each NP state and knowing the projected proportion of patients and the duration of time in each state, one can predict the costs of care.

There are limitations to the current study. First although an inception cohort study is well suited to document NP events occurring early and likely due to active lupus, our study is not well positioned to detect NP events later in the disease course such as stroke and cognitive impairment from atherosclerosis and vascular dementia. Further follow-up will be required to address this. Second, SLICC is comprised predominantly of academic centres with a special interest in SLE that may not reflect community clinical practice. Third, as this is an observational study conducted in multiple international centers with annual study assessments there is potential for variability in data collection. Close communication between SLICC sites and operational rules for data collection and attribution have been implemented to keep this to a minimum. Finally, the complexity of a reversible multistate model to predict long-term probabilities of state occupancy, requires the use of parametric assumptions for transition rates, generally that of constant rates over time. However, variation over time as reflected by movements between the states is the primary focus of the modelling.

Despite these limitations our study provides a comprehensive overview of NP events, their attribution and outcome in the first decade following the diagnosis of SLE in a representative group of SLE patients. Multistate modelling is a potential outcome 
measure in clinical trials of NPSLE. Future studies will examine in detail the predictors of transition between the NP states and the economic costs associated with NPSLE.

\section{Acknowledgements}

The Corresponding Author has the right to grant on behalf of all authors and does grant on behalf of all authors, an exclusive license (or non-exclusive for government employees) on a worldwide basis to the BMJ Publishing Group Ltd and its Licensees to permit this article (if accepted) to be published in Annals of the Rheumatic Diseases and any other BMJPGL products to exploit all subsidiary rights, as set out in our license (http://group.bmj.com/products/journals/instructions-for-authors/licence-forms.

\section{Funding:}

Core funding for this investigator-initiated study was provided to Dr. John G. Hanly by the Canadian Institutes of Health Research (grant MOP-88526).

\section{Other sources of funding supported activities at individual SLICC sites:}

Dr. Sang-Cheol Bae's work was supported in part by_NRF-2017M3A9B4050335, Republic of Korea.

Dr Caroline Gordon is supported by Lupus UK, Sandwell and West Birmingham Hospitals NHS Trust and the National Institute for Health Research (NIHR)/Wellcome Trust Birmingham Clinical Research Facility. The views expressed are those of the authors(s) and not necessarily those of the NHS, the NIHR or the Department of Health. 
The Hopkins Lupus Cohort is supported by the NIH (grant AR43727 and 69572).

The Montreal General Hospital Lupus Clinic is partially supported by the Singer Family Fund for Lupus Research.

Dr. Clarke holds The Arthritis Society Chair in Rheumatic Diseases at the University of Calgary.

Dr. Paul R. Fortin holds a tier 1 Canada Research Chair on Systemic Autoimmune Rheumatic Diseases at Université Laval.

Dr. Bruce is a National Institute for Health Research (NIHR) Senior Investigator and is supported by Arthritis Research UK, the NIHR Manchester Biomedical Centre and the NIHR/Wellcome Trust Manchester Clinical Research Facility. The views expressed in this publication are those of the author(s) and not necessarily those of the NHS, the National Institute for Health Research or the Department of Health.

Dr. Soren Jacobsen is supported by the Danish Rheumatism Association (A3865) and the Novo Nordisk Foundation (A05990).

Dr. Ramsey-Goldman's work was supported by the NIH (grants 5UL1TR001422-02, formerly 8UL1TR000150 and UL-1RR-025741, K24-AR-02318, and P60AR064464 formerly P60-AR-48098).

Dr. Mary Anne Dooley's work was supported by the NIH grant RR00046.

Dr. Ruiz-Irastorza is supported by the Department of Education, Universities and Research of the Basque Government. 
Dr Isenberg and Dr Rahman are supported by the National Institute for Health Research University College London Hospitals Biomedical Research Centre.

Dr. Lim's work was supported, in part, by the Centers for Disease Control and Prevention grant U01DP005119.

Contributors: All authors participated in the collection of research data or the data analysis or writing and critical review of the manuscript.

Competing interests: RVV has received grants from BMS, GSK, Lilly, Pfizer, UCB Pharma, personal fees from AbbVie, AstraZeneca, Biotest, Celgene, GSK, Janssen, Lilly, Novartis, Pfizer, Servier, UCB, outside the submitted work. The other authors have nothing to declare.

Data sharing statement: All data relevant to the study are included in the article or uploaded as supplementary information. 


\section{Legends for figure}

Figure 1: Reversible multistate Markovian model for observed transitions in neuropsychiatric (NP) status in patients with SLE.

Figure 2: Layered plot of trajectories of NP states recorded at study assessments, for all study patients, ordered sequentially at each assessment by NP states.

Figure 3: Time from diagnosis of SLE to onset of new and recurrent SLE neuropsychiatric (NP) events (left panel) and for non-SLE neuropsychiatric events identified by attribution model B (right panel). For recurrent events the time origin was the resolution of any previous event. The rate of recurrent SLE NP events occurred at a higher rate than first events with little difference in these rates for non-SLE events.

Figure 4: SF-36 physical component (PCS) and mental component (MCS) summary scores (mean and 95\% confidence interval) for patients in different neuropsychiatric (NP) states (global p-value<0.001). The SLE NP events and non-SLE NP events were determined using attribution model $\mathrm{B}$. 


\section{References}

1. Hanly JG, Kozora E, Beyea SD, Birnbaum J. Review: Nervous System Disease in Systemic Lupus Erythematosus: Current Status and Future Directions. Arthritis Rheumatol. 2019;71(1):33-42.

2. Hanly JG. Diagnosis and management of neuropsychiatric SLE. Nat Rev Rheumatol. 2014;10(6):338-47.

3. Ahn GY, Kim D, Won S, Song ST, Jeong HJ, Sohn IW, et al. Prevalence, risk factors, and impact on mortality of neuropsychiatric lupus: a prospective, singlecenter study. Lupus. 2018;27(8):1338-47.

4. Cook RJ, Lawless, J.F. Multistate Models for the Analysis of Life History Data. Boca Raton, Florida, USA: CRC Press; 2018.

5. Isenberg D, Ramsey-Goldman R. Systemic Lupus International Collaborating Group--onwards and upwards? Lupus. 2006;15(9):606-7.

6. Hochberg MC. Updating the American College of Rheumatology revised criteria for the classification of systemic lupus erythematosus. Arthritis Rheum. 1997;40(9):1725.

7. Gladman DD, Ibanez D, Urowitz MB. Systemic lupus erythematosus disease activity index 2000. J Rheumatol. 2002;29(2):288-91.

8. Gladman D, Ginzler E, Goldsmith C, Fortin P, Liang M, Urowitz M, et al. The development and initial validation of the Systemic Lupus International Collaborating Clinics/American College of Rheumatology damage index for systemic lupus erythematosus. Arthritis Rheum. 1996;39(3):363-9.

9. The American College of Rheumatology nomenclature and case definitions for neuropsychiatric lupus syndromes. Arthritis Rheum. 1999;42(4):599-608.

10. Ainiala H, Hietaharju A, Loukkola J, Peltola J, Korpela M, Metsanoja R, et al. Validity of the new American College of Rheumatology criteria for neuropsychiatric lupus syndromes: a population-based evaluation. Arthritis Rheum. 2001;45(5):419-23.

11. Hanly JG, Urowitz MB, Su L, Sanchez-Guerrero J, Bae SC, Gordon C, et al. Short-term outcome of neuropsychiatric events in systemic lupus erythematosus upon enrollment into an international inception cohort study. Arthritis Rheum. 2008;59(5):721-9.

12. Hanly JG, Urowitz MB, Sanchez-Guerrero J, Bae SC, Gordon C, Wallace DJ, et al. Neuropsychiatric events at the time of diagnosis of systemic lupus erythematosus: an international inception cohort study. Arthritis Rheum. 2007;56(1):265-73.

13. Hanly JG, Urowitz MB, Jackson D, Bae SC, Gordon C, Wallace DJ, et al. SF-36 summary and subscale scores are reliable outcomes of neuropsychiatric events in systemic lupus erythematosus. Ann Rheum Dis. 2011;70(6):961-7.

14. Thumboo J, Fong KY, Ng TP, Leong KH, Feng PH, Thio ST, et al. Validation of the MOS SF-36 for quality of life assessment of patients with systemic lupus erythematosus in Singapore. J Rheumatol. 1999;26(1):97-102.

15. R Core Team. R: A Language and Environment for Statistical Computing. Vienna, Austria: R Foundation for Statistical Computing.; 2015. 
16. Jackson $\mathrm{CH}$. Multi-state models for panel data: the MSM package for $\mathrm{R}$. Journal of Statistical Software. 2011;38:1-29.

17. Hanly JG, Li Q, Su L, Urowitz MB, Gordon C, Bae SC, et al. Cerebrovascular Events in Systemic Lupus Erythematosus. Arthritis Care Res (Hoboken). 2018.

18. Fanouriakis A, Pamfil C, Rednic S, Sidiropoulos P, Bertsias G, Boumpas DT. Is it primary neuropsychiatric systemic lupus erythematosus? Performance of existing attribution models using physician judgment as the gold standard. Clin Exp Rheumatol. 2016;34(5):910-7.

19. Farewell VT, Su L. A multistate model for events defined by prolonged observation. Biostatistics. 2011;12(1):102-11.

20. Jackson CH, Su L, Gladman DD, Farewell VT. On Modelling Minimal Disease Activity. Arthritis Care Res (Hoboken). 2016;68(3):388-93.

21. Hanly JG, Su L, Urowitz MB, Romero-Diaz J, Gordon C, Bae SC, et al. A Longitudinal Analysis of Outcomes of Lupus Nephritis in an International Inception Cohort Using a Multistate Model Approach. Arthritis Rheumatol. 2016.

22. Bruce IN, O'Keeffe AG, Farewell V, Hanly JG, Manzi S, Su L, et al. Factors associated with damage accrual in patients with systemic lupus erythematosus: results from the Systemic Lupus International Collaborating Clinics (SLICC) Inception Cohort. Ann Rheum Dis. 2015;74(9):1706-13.

23. Hanly JG, O'Keeffe AG, Su L, Urowitz MB, Romero-Diaz J, Gordon C, et al. The frequency and outcome of lupus nephritis: results from an international inception cohort study. Rheumatology (Oxford). 2016;55(2):252-62. 